\title{
Xenon-inhibition of the MscL mechano-sensitive channel and the CopB copper ATPase under different conditions suggests direct effects on these proteins
}

Evgeny Petrov $^{1 \llbracket}$, Gopalakrishnan Menon ${ }^{1 \llbracket}$, Paul R. Rohde ${ }^{2}$, Andrew R. Battle ${ }^{2,3}$, Boris Martinac ${ }^{2,4}$, Marc Solioz ${ }^{1,5}$

${ }^{1}$ Laboratory of Biochemistry and Molecular Biology, Tomsk State University, Tomsk, Russia

${ }^{2}$ Victor Chang Cardiac Research Institute, Darlinghurst, Australia

${ }^{3}$ School of Biomedical Sciences, Queensland University of Technology (QUT), Brisbane, Australia

${ }^{4}$ St Vincent's Clinical School, University of New South Wales, Darlinghurst, Australia

${ }^{5}$ Department Clinical Research, University of Bern, Bern, Switzerland

*Corresponding author

E-mail: marc@solioz-scientific.ch

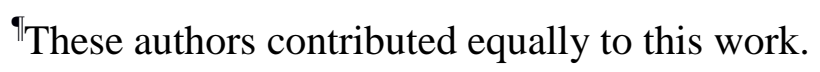

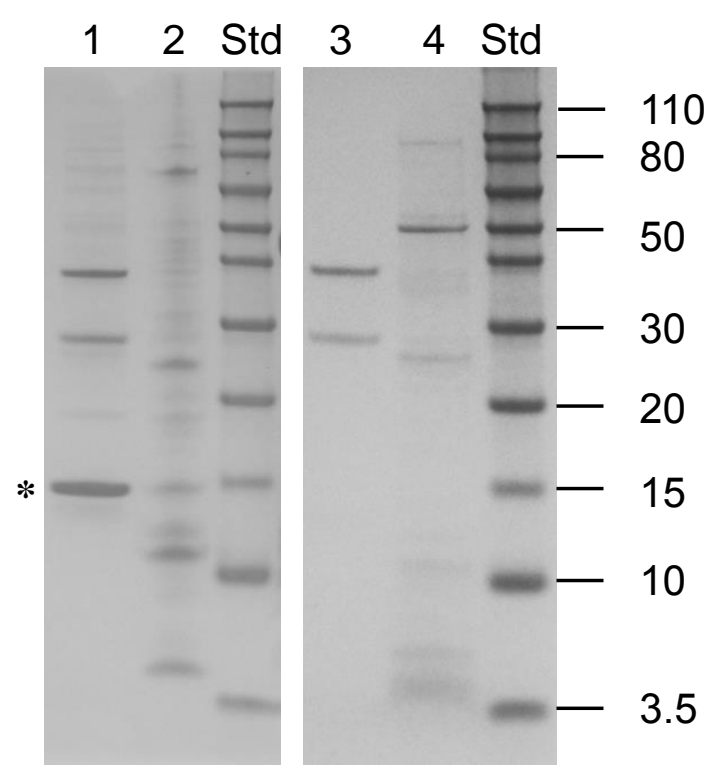

Figure A. Purification of MscL-G22E. Lane 1, approximately $2 \mu \mathrm{g}$ of MscL-G22E released from the column by $0.58 \mathrm{U}$ of thrombin; lane 2 , total fraction of plasmid-directed in vitro protein synthesis bound to the column; lane 3, $0.58 \mathrm{U}$ of thrombin used for cleavage; lane 4, unrelated sample; Std, molecular weight standards as indicated in $\mathrm{kDa}$ in the Figure. The Figure shows $12 \%$ polyacrylamide/SDS gels stained with SimplyBlue SafeStain (Novex); the asterisk indicates MscLG22E. 


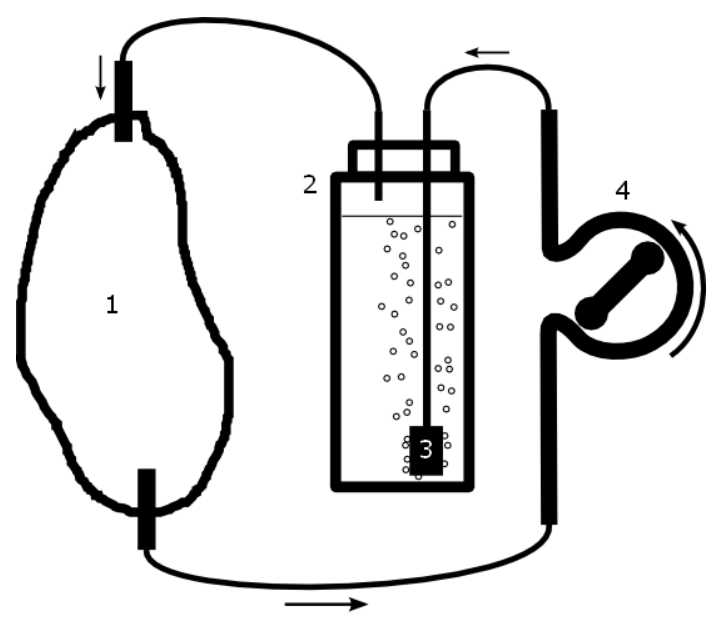

Figure B. Device for saturation of solution with xenon. 1, Plastic bag with 21 of xenon; 2, glass jar (500 ml) with recording solution; 3, gas diffuser; 4, peristaltic pump.
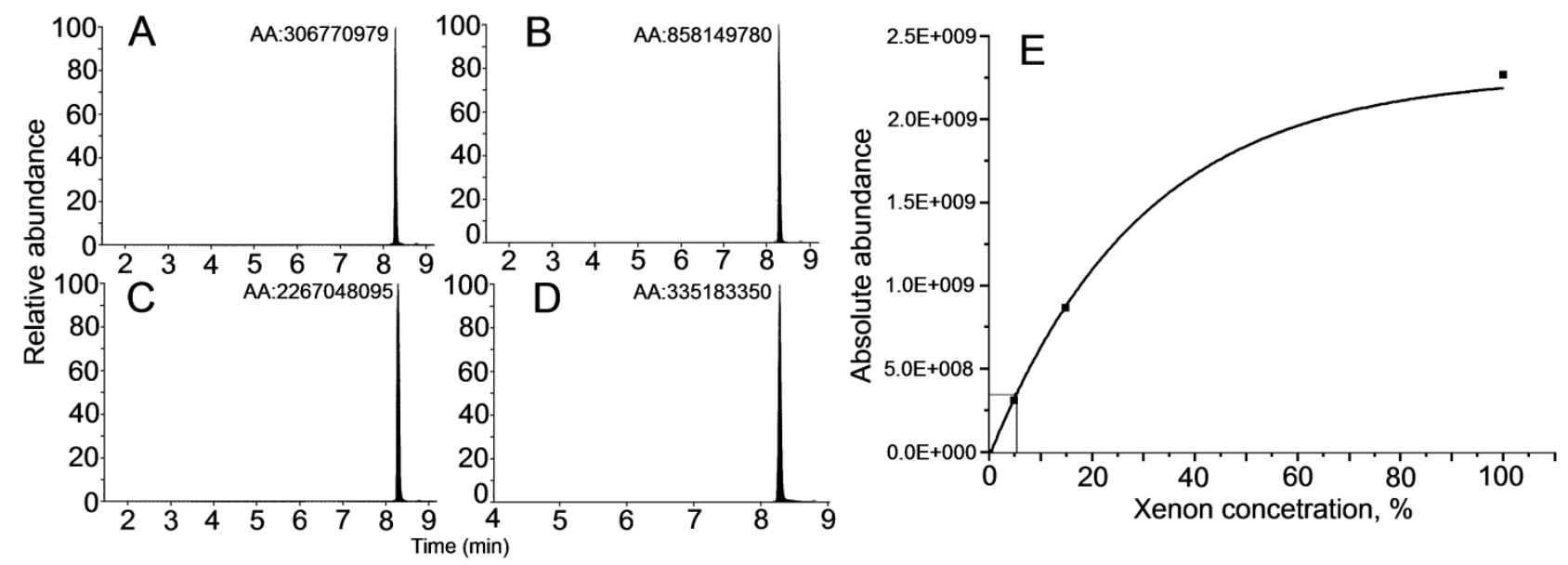

Figure C. Determination of the xenon concentration in buffers. Mass spectra of calibration solutions containing 5\% (A), 15\% (B), and 100\% (C) xenon in air, and of a saturated buffer solutions (D). E. Calibration curve for xenon measurements. AA, absolute abundance of ${ }^{132} \mathrm{Xe}$.

Table A. Protease inhibitors and composition of 100x stock solution

\begin{tabular}{|l|l|l|l|l|}
\hline Abbreviation & Chemical name & Source & $\mathbf{M}_{\mathbf{r}}$ & Per 5 ml DMSO $^{1}$ \\
\hline PMSF & Phenylmethylsulfonylfluoride & Merck 7349 & 174 & $87 \mathrm{mg}$ \\
\hline TLCK & $\begin{array}{l}\text { N-Tosyl-L- } \\
\text { lysylchloromethane }\end{array}$ & Merck 24648 & 369 & $184 \mathrm{mg}$ \\
\hline TPCK & $\begin{array}{l}\text { N-Tosyl-L-phenylalanyl } \\
\text { chloromethane }\end{array}$ & Merck 8615 & 352 & $176 \mathrm{mg}$ \\
\hline pABA & $p$-Aminobenzamidine & Sigma A7148 & 208 & $104 \mathrm{mg}$ \\
\hline
\end{tabular}

${ }^{1}$ DMSO, dimethylsulfoxide 


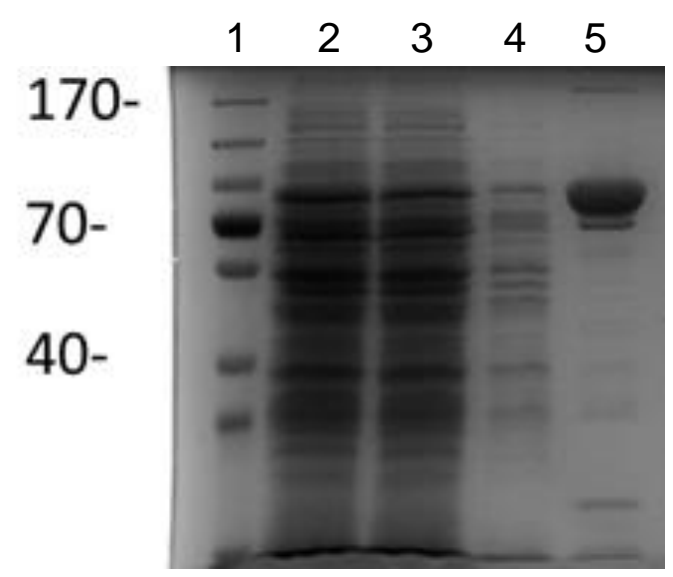

Figure D. Purification of CopB. Lane 1, molecular weight markers with 170, 70, and $40 \mathrm{kDa}$ indicated to the left of the figure; lane 2, crude extract of induced cells; lane 3, Ni-NTA column flow-through; lane 4, column wash with $10 \mathrm{mM}$ imidazole in JD-buffer; lane 5, elution of CopB with $200 \mathrm{mM}$ imidazole in JD-buffer. The figure shows a 10\% polyacrylamide/SDS gel stained with Coomassie blue.
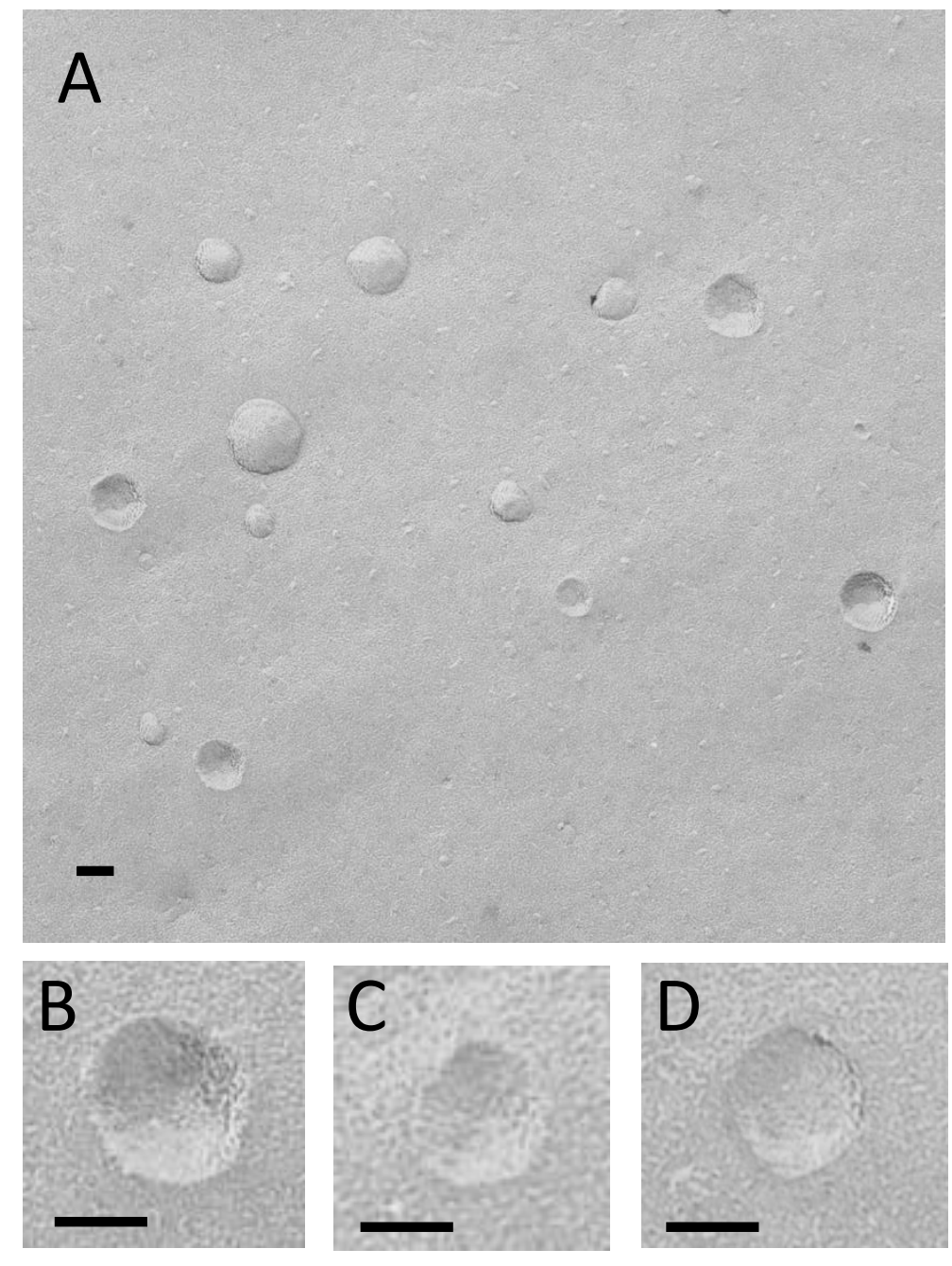

Figure E. Freeze-fracture electron micrographs of CopB proteoliposomes. (A), Lowmagnification survey of proteoliposomes with a lipid/protein weight ratio of 50, prepared by octyl glucoside dialysis as outlined in the main text. (B), (C), and (D), concave fracture faces of individual vesicles, from the preparation shown in panel (A), at higher magnification to display particles. Shadowing was from the lower right and the bars correspond to $100 \mathrm{~nm}$. 


\section{Supplementary methods}

Freeze-fracture electron micrographs of proteoliposomes were prepared by concentrating the reconstituted vesicles 10-fold by exposing them in a dialysis tubing to dry polyethylene glycol 6000 for 30- $40 \mathrm{~min}$. Less than $1 \mu \mathrm{l}$ of sample was then frozen between copper strips in super-cooled liquid nitrogen and fractured under vacuum at $-110^{\circ} \mathrm{C}$. Samples were etched for $1 \mathrm{~min}$ at this temperature, followed by cooling to $-260{ }^{\circ} \mathrm{C}$ for shadowing with $1 \mathrm{~nm}$ of platinum/carbon at an angle of $45^{\circ}$ and $20 \mathrm{~nm}$ of carbon at $90^{\circ}$. The cleaned replicas were mounted on uncoated 400 mesh grids and observed in a Phillips 420 electron microscope at $100 \mathrm{kV}$. 